\title{
Conceptual Spaces for Space Event Characterization
}

\author{
Jeremy R. Chapman*, David Kasmier ${ }^{\dagger}$, David Limbaugh ${ }^{\dagger}$, Stephen R. Gagnon ${ }^{\S}$, John L. Crassidis ${ }^{\mathbb{I}}$, James Llinas ${ }^{\|}$, \\ Barry Smith**, and Alexander P. Cox ${ }^{\dagger \dagger}$ \\ University at Buffalo, The State University of New York, Amherst, New York, 14260-4400 \\ CUBRC Inc., Buffalo NY 14225
}

\begin{abstract}
A method for space event characterization using the framework of conceptual spaces will be illustrated in this paper. The focus of this paper is on estimating the likelihood of a collisions between space objects. We hope to develop an approach for anticipatory decision support for space operators so that preventative actions can be taken based on the assessment of relative risk. The conceptual spaces approach will draw on fusion of both hard and soft data into a single decision framework. The fusion-bases approach presented in this paper for decision support extends the conceptual spaces framework with a set of ontologies by drawing on the space domain ontologies, a large system of ontologies designed to support aspects of space situational awareness. This framework is coupled with a mathematical optimization approach, providing a quantitative basis for ranking potential for collision across multiple satellite pairs. The goal is to provide the broadest possible information foundation for critical assessments of collision likelihood
\end{abstract}

\section{Introduction}

$\mathrm{T}$ HIS paper is written to utilizes the conceptual spaces (CS), presented in [1] framework for the fusion of different types of data for anticipatory decision support for space operators. The overall objective for this data fusion for space events is to increase Space Situational Awareness (SSA). Although space is currently not considered a hostile zone the threat of a orchestrated intentional satellite collision caused by hostile parties should be prepared for. As the number of threats in space increases it is becoming progressively more difficult to identify threats in real time. Throughout this paper satellites assumed to pose a threat will be referred to as Red satellites, their assumes targets that we want to protect will be referred to as Blue satellites. Our methods measure and rank the threat of a kinetic kill event, which in this context means an intentional act of a physical collision between a pair of satellites that is carried out with the intention of damaging or destroying the satellite that forms the target.

In essence this paper seeks to utilizes conceptual spaces to mimic human decision making to assess which satellites are at risk for an attack. When humans make decisions we utilize an abundant amount of potentially helpful information; including measurable data, contextual data, and our own human intuition. For space events this information includes observation data that is derived from instruments such as radar and telescopes used to determine a satellite's position and photometry information. Measurable information of these natures are examples of hard data. Other information about a satellite used in decision making that is not derived directly from instrument measurements but instead from human perception, judgment, and analysis, is commonly reference to as soft data and is often in textual form. Some examples of soft data include social political aspects of the relationship between Red and Blue satellites country of ownership as well as a satellites component systems and thruster type. Human derived information is not easily quantified, and its uncertainty is also difficult to quantify. This paper presents a method utilizing conceptual spaces to illustrate that both types of data can be exploited to establish the relative threat posed by a given Red to a given Blue satellite.

Data fusion presents many challenges throughout the data fusion community. Many work has been done in the past for data fusion to overcome these challenges. Complex terminology of and concepts need to be combined in a organized and systematic approach. Another challenge is how to deal with the complex physics of a satellite-pair

\footnotetext{
*Graduate Student, Department of Mechanical \& Aerospace Engineering. Email: jeremych@buffalo.edu, Student Member AIAA

† Postdoctoral Researcher, Department of Philosophy

†Lecturer, Department of Philosophy

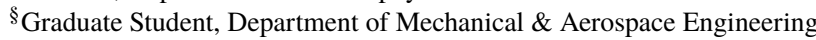

IISUNY Distinguished Professor and Samuel P. Capen Chair Professor, Department of Mechanical \& Aerospace Engineering. Email: johnc@buffalo.edu. Fellow AIAA.

" Professor, Department of Civil Engineering

** Julian Park Distinguished Professor, Department of Philosophy

${ }^{\dagger}$ CUBRC Inc.
} 
collision. The physics that describe the likelihood of a collision is described by Lambert's Problem, which is the problem of determining the orbit between endpoints when the two positions vectors and the time of flight between each satellite's position are known [2]. Another challenge that will be discussed in this paper is how to address the underlying mathematics of conceptual spaces. Conceptual spaces by themselves were originally created as a cognitive model for information fusion and did not detail the underlying mathematics. For conceptual spaces to be utilized as a tool a mathematical framework needs to be implemented. These challenges will be address in detail throughout this paper.

\section{Complex terminology}

In regards to the challenge presented by the complex terminology the main strategy to produce an organized and systematic approach is to extend the conceptual spaces framework with a set of ontologies. Ontologies make information more accessible and discoverable by tagging, and thereby semantically enhancing, items of data in large information systems. Ontologies increase interoperability between people, organizations, systems, and machines, thereby making stored date more findable and analyzable by both humans and computers. The ultimate goal of ontologies are to reduce the effort required to turn collected data into information accessible to, and supportive of, effective and timely decision making. One example of how terminology can be complex is with the word vessel. If someone was to think of a vessel in regards to a space mission they would think of a space vehicle. However, if one was to consider vessel in an anatomy sense a blood vessel would come to mind. This is an example of how two identical words can have different meanings and how using the appropriate ontology can help distinguish the proper meaning. Ontologies are defined for present purpose as a controlled vocabulary of terms that are organized hierarchically and curated by experts.

Different domain ontologies have been created for many different purposes such as Avionics Analytics[3], Military [4], and Supporting domain ontologies. For this work, data is tagged using the Space Domain Ontologies (SDO), which is a set of modules that inherits from the Common Core Ontologies (CCO). The SDO have been developed over the course of 5 years by CUBRC Inc. with input from researches at the University at Buffalo [5]. Space Domain Ontologies use international standard ISO/IEC 21838-2 [6], and the Basic Formal Ontology (BFO), [7] as the top-level ontology. An illustration of the structure of ontology and how the SDO fit into this structure can be seen in Figure 1 . The ontologies provide the semantics required to represent both soft and hard data in a structured linked knowledge based enabling fusion of data from multiple sources and in multiple formats.

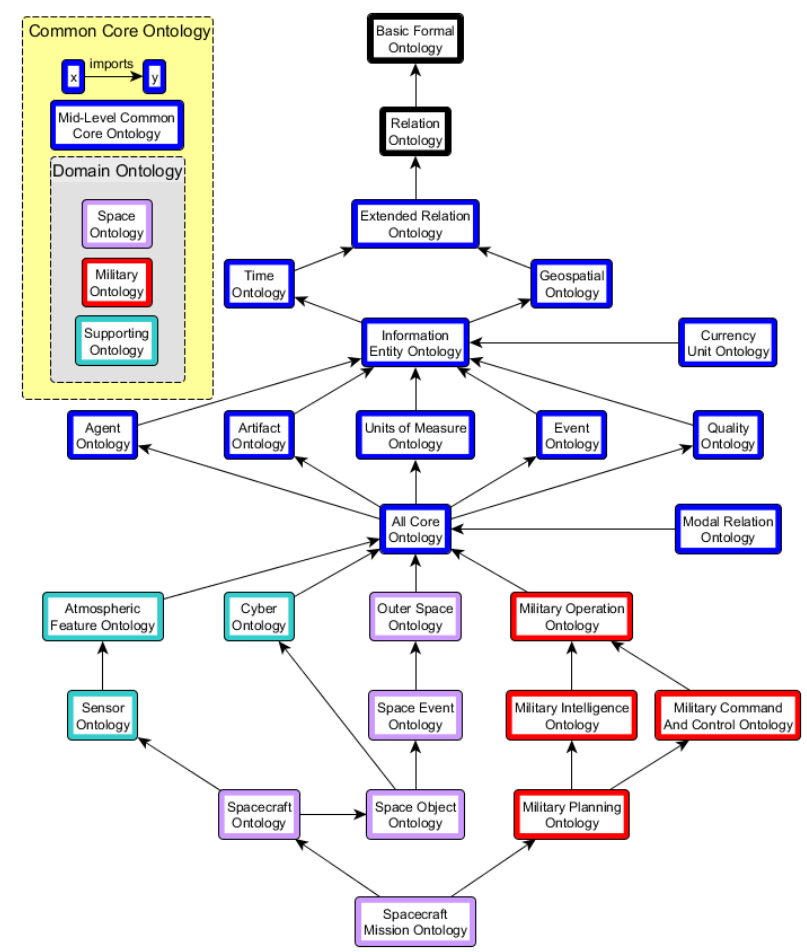

Fig. 1 Space Domain Ontologies Structure 
The Space Domain Ontologies currently contains more than 800 classes that represented entities of a wide range of different types. Space object data is aligned with classes and relations in the Space Object Ontology (SOO) and stored in a dynamically updated Resource Description Framework (RDF) triple store. The RDF can be queried to support SSA and the needs of the spacecraft, ground operators, and other users. For each type of entity in the domain the ontology provides a definition, a classification, and relational links to other entities. The ontology represents the reality and complexity of the entities in the space domain.

Once ontologically structured, data is available to be utilized for any number of applications. The fundamental background idea is that for data to be unambiguous across all applications, it must be properly represented in the ontology. For the case of this paper the primary application is to the conceptual spaces model that is being created for space event characterization.

Conceptual spaces, originally presented by Gärdenfors's [1], are meant to combine aspects of symbolic (rule based) [8-10] and associationist (feature based)[11, 12] concept representation. A conceptual spaces model is meant to be a geometric representation of how humans understand concepts. Originally CS were based on a theory of human concept possession, however its geometrical approach to similarity and differences is applicable to any and all dimensions of reality, including in this case threats to satellites. One important aspect is that we are not using concepts in people's minds but instead of certain types of objects, events, and qualities in the world. Specifically for this paper we are interested in Satellites, in Kinetic Kill events, and in Threat values, and we apply a CS model to these types of entities.

The most basic building blocks of CS representations are called quality dimensions, for example hue, saturation, and brightness in the color domain or pitch, timbre, and loudness in the domain of musical tones see Figure Figure $2 \mathrm{a}$ A dimensions is essentially an aspect or feature that has a measurable extent of some kind. The fundamental role of dimensions is to build up the domains that are needed to represent a concept. A domain can be defined as a set of integral dimensions that are separable from all other dimensions. The dimensions of the color domain, also referred to as the color space, as mentioned previously, are hue, saturation, and brightness as seen in Figure $2 \mathrm{~b}$ Also with a single domain dimensions are mutually dependent of each other, meaning one cannot exist without the existence of the other. For example if we are trying to define a color we would not know what color it is unless we know values for its hue, saturation and brightness. However to identify a color we wouldn't know anything about its pitch, timbre, or loudness as these are dimensions of the sounds domain and do not help us identify the color. The fundamental reason for decomposing a concept into domains is the assumption that your concept can be assigned certain properties within the domain that is independent of other properties.

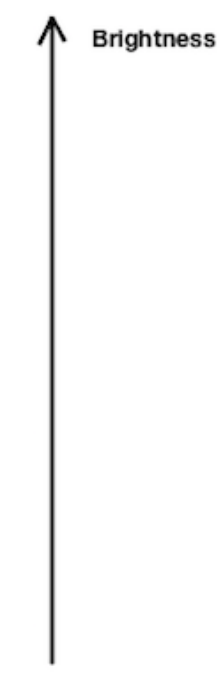

(a) Dimension

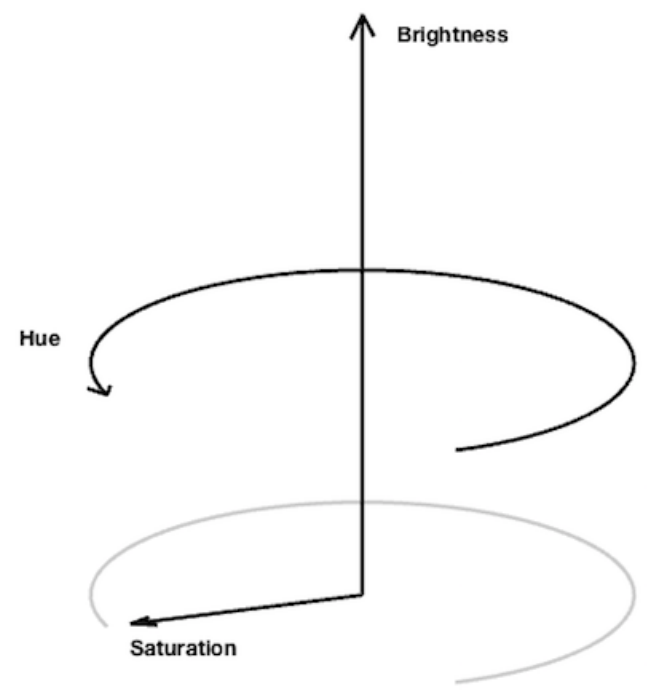

(b) Domain

Fig. 2 Characteristics lying within a single domain of a CS model

If we break down a single domains into smaller sections then we can start to define properties, which is represented 
in the CS model as a convex region of a domain space. If for example we were looking for the color blue it would be represented by a certain set of values from each dimension within the color domain. As we can see in Figure 3 the colors on the left side represent blue. The color blue would be bounded by constraints on the hue, brightness and saturation. We can relax or constrict our constraints on the property to make it more or less specific. One may say that true blue is only represented by a single point in the color domain while others would alway for a range of lighter to darker blues to all be classified as blue.

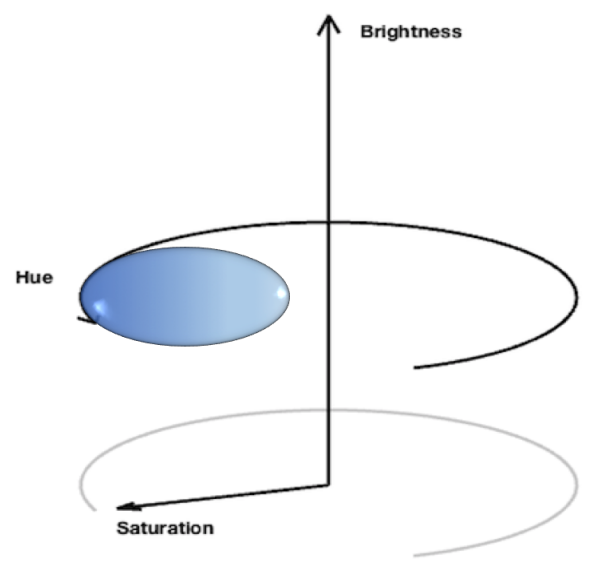

Fig. 3 Properties within the Color Domain

When building a CS model different domains will be examined. In CS there is a distinction between properties and concepts, properties are represented by a single domain while a concepts may be based on multiple domains. The unique characteristic of concepts compared to properties is that concepts span several of the domains. This is closer to how a human would identify objects, events, and qualities in the world, as knowing only one property such as its color generally isn't enough knowledge to know what is being described This distinctions between properties and concepts is not drawn within traditional symbolic or associationist representation models, this provides an added benefit to the CS approach. Concepts can have associations between multiple domains, such links between domains is known as cross-domain property associations and are useful for building concepts. For example, see Figure 4 with an apple the color and texture domains are usually associated. If an apple is brown it will generally be wrinkly however if an apple red or green it is usually smooth. The apple that is being observed is an individual object and is represented by a point in the CS model. An object that is being observed will hold a single value in each of the dimensions across all of the domains. We can have several objects such as an apple that is red and smooth or an apple that is brown and wrinkled. Each apple is a different object and each represents a point in the CS. If we have a CS model that was built with domains and properties to represent the concept of an apple then is should be able to identify both of these objects.

A CS model can be constructed to identify any sort of entity, including material objects like apples and satellites, but also events, dispositions, qualities, and relations. For example, an event, such as a station keeping maneuver of a particular satellite would have would be represented, where dimensions such as duration, location, orbital path, angular velocity, and so on has properties and cross-domain properties that indicates a station keeping maneuver. If the same CS model has properties and cross-domain properties to identify and orbital maneuver it would be able to uniquely distinguish a station keeping maneuver from and orbital maneuver.

Following the line of thinking employed for various association schemes in the fusion community, similarity between two objects or events increases as a function of the number of features they have in common and of the proximity of measurable values of those features. In our framework dimension values can be treated as independent and the distance between values of an observed quality and a specified ideal or canonical value is sufficient to capture how similar an observed object or event is to that ideal. Therefore, similarity or associability should be a function of shared dimensions and interdomain correlations. The next step in working with CS models is to quantify the above representation so that mathematical analysis can be exploited to provide the quantitative data needed to rank likelihoods of an event occurring. The CS model is semantically enhanced with ontology terms that represent measurable entities.

The measurement value of a given CS quality is tagged with the CCO term Measurement Information Content entity (M-ICE). M-ICE is defined in the CCO as "Descriptive Information Content Entity that describes the extent, 


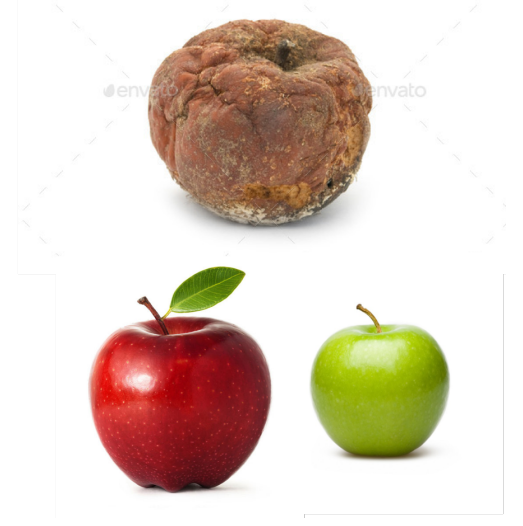

Fig. 4 Cross-Domain Property Associations

dimensions, quantity, or quality of an entity relative to some standard". Our CS model uses ontologically structured classes of measurable entities for its quality dimensions and structured measurement data in the form of M-ICEs, to tag the results of its calculations. This allows the ontology to represent various CS qualities of space objects and events and their connections and thereby to disambiguate quality dimensions from one another.

\section{Complex Physics}

The second challenge that will be discussed that arises in this data fusion problem involves the complex physics of a satellite collision between two satellites. In order for a kinetic kill event to occur, a thrust maneuver must be performed so that the targeting satellite is on a collision course with the satellite that is targeted. The physics of the satellite-pair collision likelihood is described, as mentioned previously, by Lambert's Problem, which is the problem of determining the orbit between endpoints when the two position vectors and the time of flight between each satellite's position are known. Lambert's problem is the two-point boundary value problem for the differential equation seen in Equation 1 associated with restricted two body dynamics. In essence this is the problem of finding an orbit around a primary body, in this case earth, that passes from one point to another point in a specified amount of time. Four our situation the first point will be the original starting point of the Red satellite and the ending point will be the location of the Blue satellite propagated forward to account for the time of flight required between the two satellites.

$$
\ddot{\ddot{r}}=-\mu \frac{\hat{r}}{r^{2}}
$$

If we view Figure 5 we are trying to find determine the amount of thrust, $\Delta v_{1}$, required between an original orbit represented by position vector one, $r_{1}$ and an orbit represented by position vector two, $r_{2}$. The resulting impact velocity, $\Delta v_{2}$ will determine the force of the impact. Lambert's problem has no closed form solution and must be solved by an iterative method. There are many solutions to Lambert's problem including Gauss's solution[2], Battin method [2, 13], and universal variables[2, 14].

Lambert's problem was named after Johann Heinrich Lambert because he was the first person to pose the and solve the problem, the original solution was formulated in 1761[2]. Gauss's original solution was developed to rediscover the pseudo-planet of Ceres. His method was derived in 1801 and didn't require any range information. Gauss was able to solve the orbit problem however Bate, Mueller and white indicated that the derivation of Gauss's solution is long [14]. Gauss solution is also limited by the type of orbit transfer and the spread between the vectors that is allowed[2]. Battin's method was later developed by Richard Battin in 1987 his technique was more robust and doesn't suffer from the difficulties of a $180^{\circ}$ orbit transfer that most solutions have difficulty with. The universal variable algorithm presented by Bates, Muller, and White [14] uses a iterative scheme to find the universal variables.

Once Lambert's problem is solved information about the required thrust and the impact velocity will be obtained. This information is useful in the understanding of a satellite collision. In order to maximize the amount of damage that is obtained from a collision the operator of the Red satellite will want to maximize there impact velocity. 


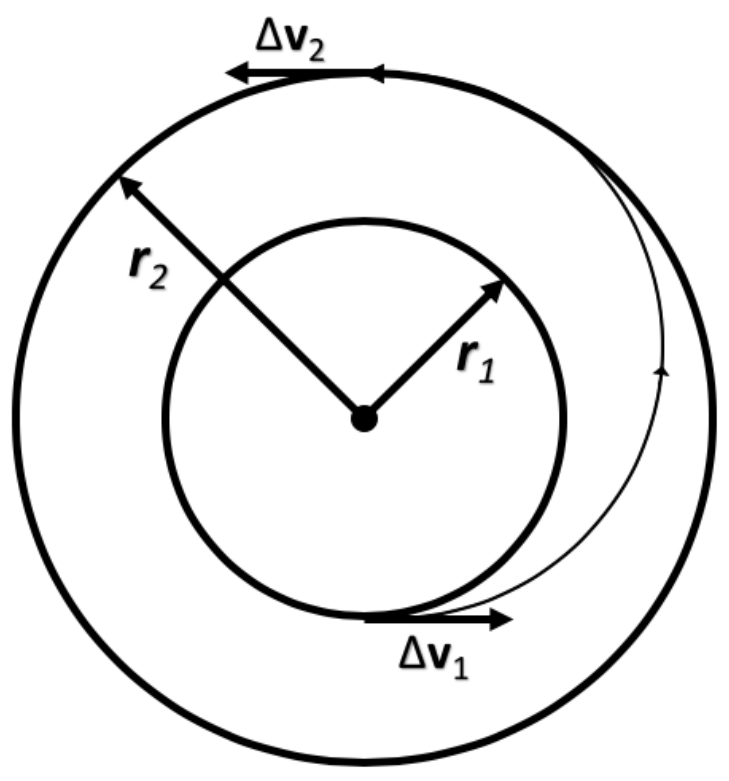

Fig. 5 Orbit Transfer

\section{Mathematical Framework for Conceptual Spaces}

Conceptual spaces gives a geometric representation of how humans understand concepts. In conceptual spaces representation there are only a few mathematical notions, such as convexity of geometries, distances between points, and similarities based on these distances, for the most part Gäredenfors leaves the creation of a mathematically representative model to other. Some work that has been done on the modeling CS mathematically can be seen in [15- -20]. This paper utilizes Holender's Conceptual Spaces - Mathematical Programming Hybrid model presented in [19, 20] in order to describe the underlying mathematics that represents CS. Mathematical programing, also known as optimization, is defined as the action of making the best or most effective use of a situation or resource.

In the optimization community optimization is better stated as a means by which one minimizes or maximizes a function over a set of constraints. A set of constraints, also known as a constraint set, represents attributes of the problem that are either feasible or not feasible. Take for example the vehicle routing problem in which the problem ask "What is the optimal set of routes for a fleet of vehicles to traverse in order to deliver to a given set of customers?". Some constraints that would need to be considered is the range of the vehicle, the drivers work day, that the driver needs periodic breaks, along with other constraints. All of the constraints, known as the constraint set, for the problem combine together to generate a region that satisfies the problems constraints, known as a feasible set wherein all the possible solutions to the problem exist. The functions that we are typing to minimize or maximize is known as the objective function and can then be optimized over the feasible set to find the best solution.

There are many types of optimization problems that can be used to solve different problems. some of the most common type of optimizations problems are known as linear programing (LP) problems[21]. The general form of linear programming is to minimize a linear objective function subject to linear constraints. Stated in standard form as,

$$
\begin{array}{r}
\min c^{T} x \\
\text { s. t. } A x=b \\
x \in S
\end{array}
$$

In the above form $x$ is a vector of unknown variables that we are trying to minimize also known as the decision variable, $c$ is known as the cost vector, $b$ is a vector, $A$ is the constraint matrix, and $S$ represents the set that the decision variable belongs to. In general the constraint matrix does not need to be square meaning the problem is underdetermined and thus the problem is not easily solved. A more special form of linear programing is integer linear programming (ILP) in which all of the variables are restricted to take on integer values. If only some of the variables are restricted to take on integer values then this problem becomes a mixed integer linear programming (MILP) problem [22]. 
Holender references two different types of conceptual spaces with different properties that must be considered for mathematical modeling. The first is single domain conceptual spaces in which each concept contains only one domain and one property within the domain. This is uses this as a stepping stone to generate more practical mathematical models. The second is complex conceptual spaces which have more then one domain and one or more property within each domain. Complex conceptual spaces are more common then single domain conceptual spaces. Holender also divides his mathematical models into two different subsections for single and multiple observations.

For the purpose paper we are mainly interested in complex conceptual spaces with a single observation. This work could be later expanded to include multiple observations. When dealing with complex conceptual spaces model there are two aspects that must be consider, these two aspects are, do the observed properties of the object we are examining exist within the concept and do cross-domain properties associate with one another exist in the observation the same way they do within the concept.

In order to set up the optimization problem for conceptual spaces we must first define a set of property combinations, for domain $i$ and properties $j$, that can be observed together to represent a specific concepts,

$$
F=\left[\left(i^{1}, j^{1}\right),\left(i^{2}, j^{2}\right), \ldots,\left(i^{n}, j^{n}\right)\right]
$$

The optimization problem is to now find the best $F$ based on a cost function, or to find the similarities between an observed object and each property defined in the concept. Stated mathematically as follows,

$$
\max \sum_{(i, j) \in F} s_{i j}
$$

Where $s_{i j}$ is the similarity of property $j$ from domain $i$. Finally we need to take into account the fact that not every decision variable, $x_{i j}$ needs to be taken into account therefore,

$$
x_{i j}=\left\{\begin{array}{l}
1, \text { if property } j \text { from domain } i \text { is considered } \\
0, \text { otherwise }
\end{array}\right.
$$

Next we can set up the following objective function that represents the observed object and the following constraint set.

$$
\begin{aligned}
& \max \sum_{i} \sum_{j} s_{i j} x_{i j} \\
& \text { s.t. } \sum_{j=1}^{n_{i}} x_{i j}=1 \forall i \\
& x_{i j}+x_{i^{\prime} j^{\prime}} \leqslant 1 \forall\left\{(i, j),\left(i^{\prime}, j^{\prime}\right)\right\} \in F \\
& x_{i j}=0 \text { or } 1 \forall i, j
\end{aligned}
$$

In this model presented the objective function represents the observed object and is maximized such that the results model conceptual spaces appropriately. It's important to note that most concepts can have multiple properties that are possible within the same domain. For example with an apple, it can either have the property of being red, green, or brown within the color domain however a green apple is no less an apple then a red apple.The constraint set consisting of equality an inequality constraints are what form the representation of the feasible region of the concept. The equality constraints represents only one property from each domain. The inequality constraint set represents the association between cross-domain properties. The solution gives an optimal value that can be normalized by the number of domains in the concept representation to obtain the similarity between the concept and the observed object.

\section{Building of Conceptual Spaces Model}

Since the overall goal of this paper is to utilized CS modeling for data fusion to increase SSA we must now identify the type of entities that we are trying to represent. In order to identify the objects, events, and qualities associated with the threat posed by a given Red-Blue pair of satellites, we first need to identify and define the domains and dimensions that are needed to capture the concept of relevant threat to the satellite. More specifically still, we focus on the threat a given Red satellite poses to a given Blue satellite with respect to a potential kinetic kill attack. The strength 
of a threat determines, in conjunction with the vulnerability of the threatened asset, the measurement value for that threat. Following Little E. \& Rogova L. (2006) an adversarial threat consists of three elements: Intent, Capability, and Opportunity [23]. We will illustrate how threat levels can be calculated in terms of these three elements combined with target vulnerability in such a way as to determine relative rankings of the obtaining threats for a given configuration of Red and Blue assets. The resulting four elements constitute the four domains of our CS model.

In order to asses if a particular space event is about to occur we must examines the satellites intentions, capabilities, opportunities, and vulnerabilities for the particular event that we are trying to observe. To assess the relevant capabilities, intentions, opportunities, and vulnerabilities of each spacecraft and its environment we use data about the space operational environment, including data about objects, events, qualities, relationships, dispositions, agents, and information that has been tagged with the SDO.

\section{A. Intent}

The first domain that will be discussed is intent, which involves the various dispositions - the beliefs, desires, habits, plans, and attitudes - that constitute the propensity of an adversary to initiate a hostile action. In BFO a disposition is a realizable entity, by which is meant an entity that can (but need not) be manifested in some process - as the disposition of fragility is manifested in the process of breaking through fragmentation. A disposition is borne by a given material entity in virtue of the latter's physical make up and its realization occurs when and because its bearer is in some special triggering circumstance.

For our initial application we take political tension to be one indicator of intent and thus our first dimension of intent, so that if the tension is high between the owners, respectively, of a Red and a Blue satellite, then they will have a greater disposition to hostile action, and thus pose a higher threat to each other. For this analysis we will assume a hypothetical political tension level between ownership of Red and Blue satellites. However, in a real-world application political tension can be measured by using one or more of the existing geopolitical risk indices, including the Geopolitical Risk Index, the World Economic Forum annual global risk report, the Heidelberg Institute for International Conflict Research conflict barometer, and the EU Global Conflict Risk Index [24-27].

The second dimension for the domain of intent in our model will be based on a measurement of the rate at which level of tension is rising or decreasing. It will be assumed that rising tension will be more threatening than decreasing tension. Both of these dimensions will be measured on a scale of 0 to 100 . For the case of political tension 0 will be low and 100 will be considered high. For level of rising or decreasing tension 0 will indicate the highest level of decreasing tension and 100 will indicate the highest level or rising tension. The middle point of 50 will be the neutral point where tension is neither rising nor decreasing.

\section{B. Opportunity}

The second domain in our CS model is opportunity. Relevant types of opportunities in the space environment include spatiotemporal opportunities, geopolitical opportunities, and physical affordances. For example, the distance between two satellites can present an opportunity to successfully exercise a thruster capability and move to intercept, a legal restriction can limit the country of ownership's political opportunities to initiate a kinetic kill, and a physical barrier or avoidance defense technology may limit the collision capabilities afforded to an attacking satellite.

One dimension in this domain can be determined by the impact velocity. An intercept opportunity can be determined by solving Lambert's problem as mentioned previously. Given the solution, we can then see the impact velocity that will occur if a kinetic kill were to occur. There is a direct correlation between the impact velocity and the amount of energy behind a kinetic kill attack, and consequently, the resultant severity of the attack. This entails that the times at which maximum impact velocity of a Red satellites is achievable correspond to the best chances for a kinetic kill attack to succeed. Impact velocity can thus be used as an indicator of better or worse spatio-temporal opportunities to attack.

For purposes of intercept opportunity analysis Lambert's problem is solved for a pairs of points which describe the positions of given Blue and Red spacecraft in the near future. For any choice of Blue and Red position along their respective orbits, Lambert's problem can be solved to determine the required change in velocity in order to bring about an interception. Iteratively solving for all possible pairs gives a description of the intercept possibilities and an indication of the most desirable intercept opportunity over the analyzed time period. This analysis is similar in many ways to the "porkchop plots" that are used to determine interplanetary maneuver windows as described in [28, 29]. Porkchop plots illustrating impact velocity over a given period of time for a later intercept time can be seen in Figure 6.

As mentioned previously one dimension that can be derived from such plots is: value of impact velocity. Opportunity is highest for the satellite that can cause the highest impact velocity and thus the most damage to the Blue asset. This 
dimension is therefore used in our model to measure impact opportunity. One way to yield a salient measure is to identify the highest global maximum among all pairs and the global minimum among the pairs. We can then identify what percentage of each individual pair falls within $20 \%$ of the difference between the global maximum to the global minimum from the global maximum. As we can see in the Figure 6 the highest global maximum, from plot $b$, is 12.0452 and the lowest global minimum, from plot b, is 3.1161. Thus, we are looking for what percentage of the impact velocities falls above 10.2594 .
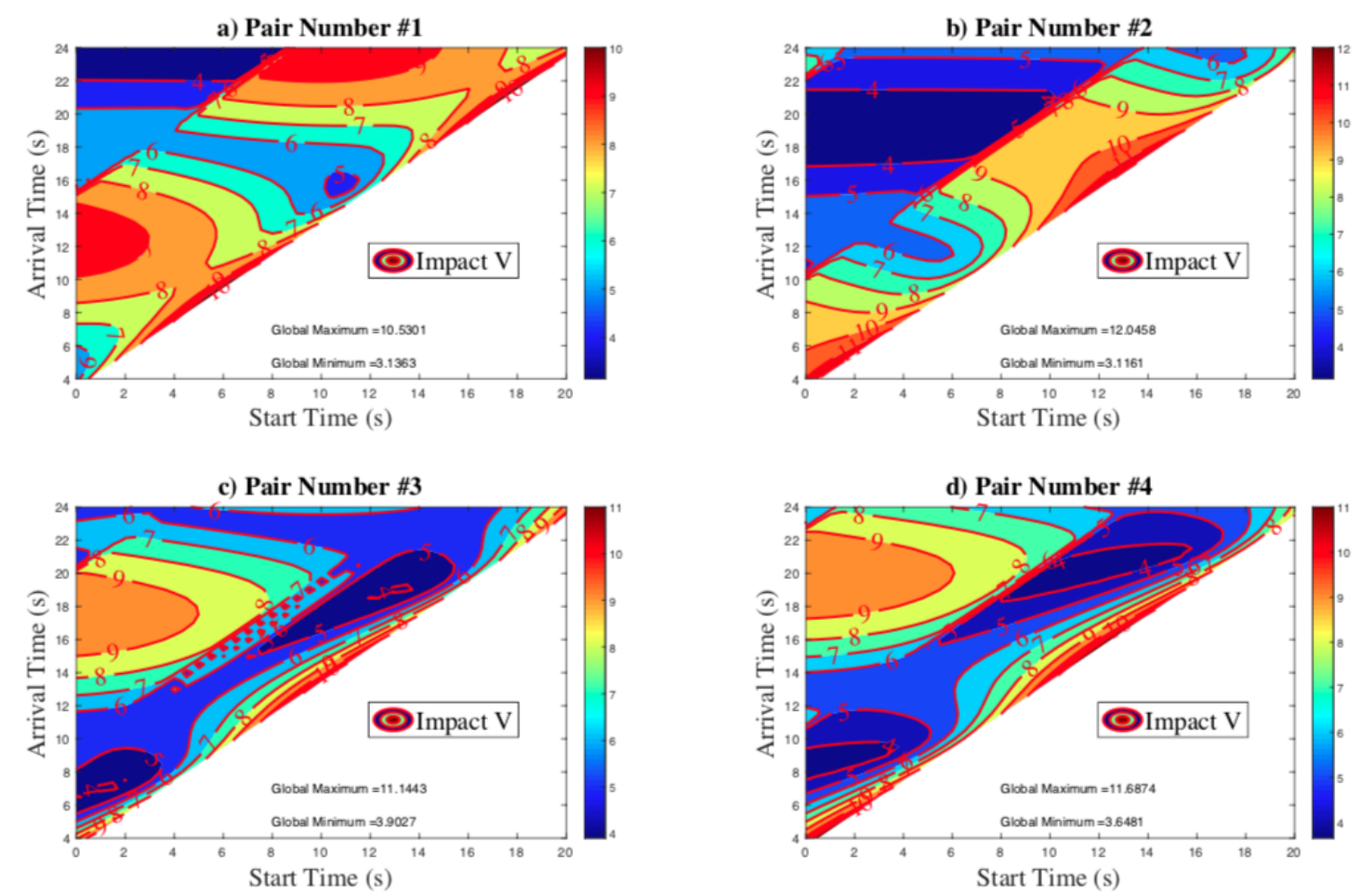

Fig. 6 Impact velocity

\section{Capability}

The third domain in our CS model of threat is capability. A capability is a disposition whose realizations are of specific interest to some person or group of people, for example the capability of a sensor to scan an area of view. A realization is of interest for example when it provides a benefit or when it is prescribed by a plan or intent. Of interest here are capabilities of a spacecraft and its parts, including component systems and subsystems. By identifying the type of spacecraft and the systems and subsystems of that spacecraft, we can identify the sorts of capabilities possessed by that spacecraft. Information gained from human-derived or semantically-derived information sources for example from [30] or from publicly available intelligences can provide measurement values that inform the extent of the spacecraft's capabilities and from this we can determine how the spacecraft can be used.

The capability of a spacecraft can be measured along multiple dimensions. The two dimensions utilized in our CS model are the specific impulse value, $I_{s p}$ of the satellite's main thruster, and the fuel mass. The force produced from the thruster, $F_{\text {thrust }}$, is derived from the product of the thruster's specific impulse[31], $I_{s p}$, the mass flow rate, $\dot{m}$, and the acceleration due to gravity, $g_{0}$, therefore,

$$
F_{\text {thust }}=g_{0} \times I_{s p} \times \dot{m}
$$

The mass flow rate is measured in $\mathrm{kg} / \mathrm{s}$ and is an indicator of how quickly fuel is being expelled. Since satellites must carry all of their fuel with them mass flow rate is equivalent to the change in the satellites mass, assuming that nothing else is being lost from the satellite. The fuel mass onboard a satellite is thus an indicator of how much force it 
can potentially produce. This gives the fuel mass dimension particular importance in our CS model. However, account must be taken also of the fact that, other things being equal, the fuel mass on a smaller satellite will obviously be smaller than fuel mass on a larger satellite. This means that we should look rather at the percentage of fuel mass that is left aboard a satellite. A satellite with zero percent available fuel mass will be completely non-threatening; a satellite with 100 percent of available fuel mass will be at its most capable and thus maximally threatening.

Different types of thrusters are characterized by the type of propellent that they use, which can include cold gas, solid propellent, and liquid oxygen/liquid hydrogen. Different types of thrusters have different specific impulse values (typical $I_{s}$ values for different types of propellants can be seen in Table 1 [31, 32]). The higher a satellite's $I_{s p}$ value is, the more capable it is of creating enough force for an attack. Thus, the higher its $I_{s p}$ value, the more threatening the satellite will be. We can nondimensionalize the $I_{s p}$ value to be on a scale between 0 and 100 . The 100 would represent some theoretical maximum that can be estimated by studying all types of thrusters currently being utilized. The value of zero is utilized for a satellite that is assumed to have no thrusting capabilities at all.

Table 1 Typical Specific Impulses

\begin{tabular}{cc}
\hline Propellent & Specific Impulse, $I_{s p}$ (seconds) \\
\hline Cold gas & 50 \\
Monopropellant hydrazine & 230 \\
Solid propellant & 290 \\
Nitric acid/monomethylhydrazine & 310 \\
Liquid oxygen/liquid hydrogen & 455 \\
\hline
\end{tabular}

\section{Vulnerability}

The final domain in our example model is vulnerability, given that the ranking of threats also requires an assessment of vulnerability. We define vulnerability as follows.

CCO: Vulnerability =Def. Disposition that inheres in a bearer in virtue of its being susceptible to having its otherwise reliable participation in processes of a certain type disrupted by a process of another type.

SOO: Collision Vulnerability =Def. Vulnerability that is realized by an act of collision that damages or destroys some or all of the target object's capabilities.

SOO: Kinetic Kill Vulnerability $=$ Def. Collision Vulnerability that is realized by an act of collision in which a kinetic kill vehicle is used to damage or destroy some or all of the target object's capabilities.

Vulnerability will be measured using a score using hard, soft, and contextual data derived from an assessment of the relevant Red-Blue asset pair. The vulnerability score will become more important when we start to assess multiple Blue assets. Blue assets that are more vulnerable will generate a higher threat level value in their respective Red-Blue pairing.

\section{Simulated Results}

Now that we have defined all aspects of the CS model for space event characterized we can look at an example problem. This example will produce simulated results to illustrate that Holender's conceptual spaces - mathematical programming hybrid model for data fusion is an effective method for classification of observed objects. Simulated results are based on the single observation of multiple domains for one Blue satellite and four Red satellites. Making this a single observation complex conceptual spaces model system. Each individual Red-Blue pair will represent its own unique observation.

The first step that will be taken to setup the optimization problem is to setup the objective functions. For the objective functions to be setup we must make observations for each of the four satellite pairs. As mentioned in the Section IV the objective functions represent the observed object, the values for each dimension are derived by the fusion of hard, soft and/or contextual data.

Before we can observe a value for the impact velocity percentage above $20 \%$ we must first define the starting and ending location and implement one of the solutions to Lambert's problem as mentioned in Section 5 . For this simulation satellites in geostationary orbits with starting positions show in Table 2 are selected. This information are the satellites 
position in orbital elements, this information can be obtained by a two-line element (TLE) query. For Lambert's problem we need a signal starting location and a signal ending location. The starting location will be the original location of the Red satellite and the ending location will be the propagated position of the Blue satellite. Upon solving Lambert's problems using orbital elements we get the results that were shown in Figure 6 .

Table 2 Geostationary TLE

\begin{tabular}{|c|c|c|c|c|c|c|c|c|c|}
\hline Satellite & $\begin{array}{c}\text { Epoch } \\
\text { Year }\end{array}$ & $\begin{array}{l}\text { Epoch } \\
\text { Day }\end{array}$ & $\begin{array}{c}\text { Ballistic } \\
\text { Coeffi- } \\
\text { cient } \\
\left(* 10^{6}\right)\end{array}$ & Inclination & $\begin{array}{l}\text { Right } \\
\text { Ascen- } \\
\text { sion }\end{array}$ & Eccentricit & $\begin{array}{l}\text { y Argument } \\
\text { of } \\
\text { Perigee }\end{array}$ & $\begin{array}{c}\text { Mean } \\
\text { Anomaly }\end{array}$ & $\begin{array}{l}\text { Mean } \\
\text { Motion }\end{array}$ \\
\hline Blue 1 & 2019 & 41.50219 & 0.99 & 0.0281 & 85.8604 & 0003143 & 281.4346 & 176.5500 & 1.00270858 \\
\hline Red 1 & 2019 & 40.85327 & 1.40 & 0.0561 & 301.4030 & 0004532 & 4.5185 & 175.1670 & 1.00270788 \\
\hline Red 2 & 2019 & 40.91158 & -3.04 & 0.4840 & 100.0441 & 0003346 & 201.5738 & 266.2397 & 1.00275656 \\
\hline Red 3 & 2019 & 41.01355 & -1.98 & 0.0207 & 91.2968 & 0004909 & 238.8009 & 88.0315 & 1.00274860 \\
\hline Red 4 & 2019 & 41.35327 & -3.61 & 0.0113 & 124.2627 & 0002009 & 253.4117 & 5.7531 & 1.00268760 \\
\hline
\end{tabular}

In order to develop the equality and inequality constraints that make up the constraint set there must be an understanding of what the object of the most threatening kinetic kill scenario would look like. As a reminder an object represent a point within the CS model. This point would be the theoretically worst-case scenario in each of the domains.

As mentioned previously the four unique domains that are; intent, capability, opportunity, and vulnerability. Starting with the domain of intent, the value of 100 for political tension and level of worsening tension represents the highest possible level for these two dimensions. Next, if we look at the domain of opportunity, we are looking at what percent of impact velocity about $20 \%$ of the difference between the global minimum and global maximum. A value of $100 \%$ would indicate that an attack can occur at any time and will produce a very high impact velocity compared to other satellites that are being examined. This could be due to the operator of the Red satellite repositioning the satellite for an imminent attack. Thus a value of $100 \%$ would indicate a worst-case scenario. For the domain of capability a value of 100 for the dimension of percentage of fuel left would indicate that all available fuel is still onboard the satellite. The dimension of normalized $I_{s p}$ that would also have a theoretical maximum value of 100. Finally, for the domain of vulnerability, a score of 100 for vulnerability would indicate that the Blue satellite has no protection against an attack. When we analyze different Blue assets the vulnerability score will change but since we are only analysis one Blue asset the vulnerability will be the same throughout all four pairs.

The values for each of the dimensions of each observed pair can be seen in Table 3 These values along with a theoretically perfect example are what is used to generate the objective functions seen in Equation 8 . After the objective function is set up we need to define the constraints functions that can be found in Equation 9 The constraint set was generated by taking into consideration the object of a theoretically perfect scenario along with value from observation. The constraint function can be used to identify the area of out CS model that would represent a high threat level. As we can see, these constraints span all of the different independent domains. Subject area experts should redefine constraints sets as more knowledge is gained in order to best represents the concept of kinetic kill threats. Future work should also involve including other space event concepts into the CS model so that other events can be characterized and so that events are characterized correctly. Other events that should be characterized don't necessarily need to be malicious events but could be things as simple as station keeping maneuvers or orbital repositions maneuvers. Once the objective functions and constraints functions are generated linear programming can be used to find the distance in a CS model between the observation data and our target concept of a given Red-Blue paired kinetic kill threat. The theoretically perfect example will be used to normalize the final results.

$$
\begin{aligned}
& f_{1}=20 x_{1}+20 x_{2}+1.2209 x_{3}+20 x_{4}+20 x_{5}+20 x_{6} \\
& f_{2}=80 x_{1}+80 x_{2}+5.7714 x_{3}+80 x_{4}+80 x_{5}+20 x_{6} \\
& f_{3}=20 x_{1}+80 x_{2}+1.1099 x_{3}+60 x_{4}+60 x_{5}+20 x_{6} \\
& f_{4}=80 x_{1}+20 x_{2}+1.9978 x_{3}+60 x_{4}+60 x_{5}+20 x_{6} \\
& f_{5}=100 x_{1}+100 x_{2}+100 x_{3}+100 x_{4}+100 x_{5}+100 x_{6}
\end{aligned}
$$


Table 3 Observation Parameters

\begin{tabular}{ccccccc}
\hline Pair number & $\begin{array}{c}\text { Political } \\
\text { Tension }\left(x_{11}\right)\end{array}$ & $\begin{array}{c}\text { Change in } \\
\text { Political } \\
\text { Tension }\left(x_{12}\right)\end{array}$ & $\begin{array}{c}\text { Impact } \\
\text { Velocity } \\
\text { Percentage } \\
\text { above } 20 \% \\
\left(x_{21}\right)\end{array}$ & $\begin{array}{c}\text { Fuel Mass } \\
\text { Ratio\% }\left(x_{31}\right)\end{array}$ & $\begin{array}{c}\text { Normalized } \\
\text { Specific } \\
\text { Impulse Value } \\
\left(x_{32}\right)\end{array}$ & $\begin{array}{c}\text { Vulnerability } \\
\text { score }\left(x_{41}\right)\end{array}$ \\
\hline 1 & 20 & 20 & 1.2209 & 20 & 20 & 20 \\
2 & 80 & 80 & 5.7714 & 80 & 80 & 20 \\
3 & 20 & 80 & 1.1099 & 60 & 60 & 20 \\
4 & 80 & 20 & 1.9978 & 60 & 60 & 20 \\
\hline
\end{tabular}

$$
\begin{gathered}
x_{11} \leq x_{12} \leq x_{32} \leq x_{41} \leq 80 \\
x_{21} \leq 5 \% \\
x_{31} \leq 40 \% \\
x_{32}+x_{41} \leq x_{11}+x_{12} \leq x_{11}+x_{41} \leq 160
\end{gathered}
$$

Now that the objective functions and constraint sets are established linear programming is used as mentioned in Section IV to solve the optimization problem. The results of the solution can be seen in Table 4 and the ranking of these four satellites in terms of how threatening they are, as seen in Table 5. By optimizing the objective function with the defined constraints, we are finding how close this objective function is to our desired concept. The closer the object is to our desired concept the higher percentage it will have, this percentage can be thought of as the percent of similarity that our object has with our concept. Thus the higher the percent the more threatening the object is.

From the results it can be seen that pair 2 has the closest distance between the object and the concept and is thus the most threatening of the four pairs. On the other hand pair one has the furthest distance between the object and the concept and thus has the lowest percentage of the four pairs. Pair 3 and 4 had similar results, however as we can see more individual values of pair 4 are closer to the concept then of pair 3 thus pair 4 is slightly closer to the concept then pair 3 which is represented in the results.

Table 4 Results

\begin{tabular}{cccc}
\hline Pair 1 & Pair 2 & Pair 3 & Pair 4 \\
\hline $19.67 \%$ & $61.86 \%$ & $50.55 \%$ & $50.56 \%$
\end{tabular}

Table 5 Threat Level Ranking

\author{
Satellite Ranking
}

Red 2

Red 4

Red 3

Red 1

If we were examining more Blue satellites, we could make an individual list for each. We would then be able to determine which Blue asset is at the higher threat and which Red asset produces the associated threat. 


\section{VII. conclusion}

The exploration of a synthesized approach combining the structure of the conceptual spaces modeling method with Data Association techniques and ontologically-based data and information labeling shows promise for anticipatory decision support for operators dealing with space events and behaviors of concern. Much work remains to be done in both the elaboration of the CS model for space event characterization, however these early results suggest that this multidisciplinary approach has promise for challenging SSA problems.

A conceptual spaces framework was developed using four distinct domains for the classification of space events. In this work a conceptual spaces - mathematical programming hybrid model for classification of observed objects was shown to be successful in identifying threat level for space events. The ontology allowed for a systematic way of creating dimensions and domains.

As stated earlier the objective of this paper was to increase SSA by fusing data from soft, hard and contextual data using conceptual spaces. The first domain was intent which uses a combination of soft and contextual data: the first dimension discuses in this domain was political tension which contextual in nature and derived from soft data. The second dimension discussed in this domain was the change in political tension which can be considered soft data. The second domain was opportunity. The dimension of opportunity was described by the physical location of the satellites in order to make porkchop plots by solving lambert's problem. Since these measurements are derived from sensor information it is considered hard data. For the third domain of capability, specific impulse and fuel mass are utilized. Specific impulse can be observed by either hard or soft data. If human derived intelligence information is used to determine the specific impulse then this information will be soft data. However, if this information is derived from physical sensor observations then this information will be hard data. In some cases, both hard and soft data may be used to mutually support a finding. For example, a specific impulse value can be determined through the use of both intelligence information and sensor information concerning the same satellite. In other cases one type of data may be used to disconfirm another type. If, for example, intelligence information is received about the specific impulse of a satellite thruster, and subsequently this satellite is observed breaking this value then we can disregard this intelligence information. This fusion of hard and soft data can be used to define the uncertainties in the measurements. The final domain that is discussed is vulnerability which was treated as a combination of soft, hard and contextual data.

The simulated results show that the model is capable of showing a higher threat level for Red satellites that have values closer to the area of the conceptual spaces that represents our constraints of a maximum threat. Therefore, we can show that this model is capable of identifying threat levels. However, domains and dimensions should continue to be refined and expanded to better represent human decision making. Values within the domains and how these values are defined should also continue to be reevaluated for better decision making

\section{Acknowledgments}

We would like to thank Dr. Erik Blasch (Air Force Office of Scientific Research (AFOSR)) for continuous support of this project. Also we would like to show our gratitude to Dr. Joseph Raquepas (CIV USAF AFMC AFRL/RIED) for the continuous technical support and funding on this project.

\section{References}

[1] Gardenfors, P., Conceptual Spaces: The Geometry of Thought, MIT Press, Cambridge, MA, 2000.

[2] Vallado, D. A., Fundamentals of Astrodynamics and Applications, $4^{\text {th }}$ ed., chapter and pages, p. 467.

[3] Insaurralde, C., and Blasch, E., "Veracity metrics for ontologica! Decision-making support in avionics analytics," 2017, pp. 1-9. https://doi.org/10.1109/DASC.2017.8101999

[4] Valente, A., Holmes, D., and Alvidrez, F., "Using a Military Information Ontology to Build Semantic Architecture Models for Airspace Systems,” 2005, pp. 1 - 7. https://doi.org/10.1109/AERO.2005.1559635.

[5] Cox, A. P., Nebelecky, C. K., Rudnicki, R., Tagliaferri, W. A., Crassidis, J. L., and Smith, B., “The Space Object Ontology,” 19th International Conference on Information Fusion (FUSION), 2016, pp. 146-153.

[6] ISO/IEC 21838-2, "Information technology - Top-level ontologies (TLO) - Part 2: Basic Formal Ontology (BFO)," Standard, International Organization for Standardization, Geneva, CH, Oct. 2019. 
[7] Arp, R., Smith, B., and Spear, A. D., "Building Ontologies with Basic Formal Ontology," Journal of the Association for Information Science and Technology, Vol. 68, No. 3, 2015, pp. 801-804. https://doi.org/10.7551/mitpress/9780262527811.001. 0001

[8] Anderson, J. R., and Lebiere, C. J. (eds.), The Atomic Components of Thought, Lawrence Erlbaum Associates, Mahwah, NJ, 1998.

[9] Bozack, and Amanda, Social Cognitive Learning Theory, chapter and pages, pp. 1392-1394.

[10] Ataizi, and Murat, Situated Cognition, chapter and pages, pp. 3082-3084. https://doi.org/10.1007/978-1-4419-1428-6_16

[11] Cristianini, N., and Shawe-Taylor, J., An Introduction to Support Vector Machines and Other Kernel-based Learning Methods, $1^{\text {st }}$ ed., Cambridge University Press, 2000.

[12] Hsu, C.-W., and Lin, C.-J., "A Comparison of Methods for Multi-class Support Vector Machines," IEEE Transactions on Neural Networks, Vol. 13, No. 2, 2002, pp. 415-425.

[13] Battin, R. H., An introduction to the mathematics and methods of astrodynamics Richard H. Battin., rev. ed. ed., AIAA education series, American Institute of Aeronautics and Astronautics, Inc., Reston, Va, 1999.

[14] Bate, R. R., Mueller, D. D., and White, J. E., Fundamentals of Astrodynamics, Dover Publications, New York, 1971.

[15] Aisbett, J., and Gibbon, G., "A General Formulation of Conceptual Spaces as a Meso Level Representation," Artificial Intelligence, Vol. 133, No. 1-2, 2001. doi 10.1016/s0004-3702(01)00144-8.

[16] Rickard, J., "A concept geometry for conceptual spaces," FO \& DM, Vol. 5, 2006, pp. 311-329. https://doi.org/10.1007/s10700006-0020-1

[17] Rickard, J. T., and Yager, R. R., "Hypercube Graph Representations and Fuzzy Measures of Graph Properties," IEEE Transactions on Fuzzy Systems, Vol. 15, No. 6, 2007, pp. 1278-1293.

[18] Rickard, J., Aisbett, J., and Gibbon, G., "Reformulation of the theory of conceptual spaces," Information Sciences, Vol. 177, 2007, pp. 4539-4565. https://doi.org/10.1016/j.ins.2007.05.023

[19] Holender, M., Sudit, M., Nagi, R., and Rickard, J., "Information fusion using conceptual spaces: Mathematical programming models and methods," Information Fusion, 2007 10th International Conference on, 2007, pp. 1 - 8. https://doi.org/10.1109/ ICIF.2007.4408111

[20] Holender, M. N., "Conceptual spaces as a modeling system for Information Fusion," Ph.D. thesis, State University of New York at Buffalo, 2008.

[21] Schrijver, A., Theory of Linear and Integer Programming, John Wiley \& Sons, Inc., USA, 1986.

[22] Nemhauser, G. L., and Wolsey, L. A., Integer and Combinatorial Optimization, Wiley-Interscience, USA, 1988.

[23] Little, E. G., and Rogova, G. L., “An Ontological Analysis of Threat and Vulnerability,” 9th International Conference on Information Fusion, 2006, pp. 1-8.

[24] Caldara, D., and Iacoviello, M., "Measuring Geopolitical Risk," International Finance Discussion Papers 1222, Board of Governors of the Federal Reserve System (U.S.), Feb. 2018.

[25] Franco, E. G., The Global Risk Report 2020, 15 ${ }^{\text {th }}$ ed., World Economic Forum, 2020.

[26] Geugjes, M., Linke, F., and Stüber, V., Conflict Barometer 2018, Heidelberg Institute for International Conflict Research (HIIK) e.V., 2019.

[27] Stamatia, H., Ferri, S., Ines, J., Saporiti, F., and Kauffmann, M., "The Global Conflict Risk Index (GCRI) Regression model: data ingestion, processing, and output methods 2017,", 01 2017. https://doi.org/10.2760/303651.

[28] Ishimatsu, T., Hoffman, J., and de Weck, O., "Method for Rapid Interplanetary Trajectory Analysis using $\Delta$ V Maps with Flyby Options," Journal of the British Interplanetary Society, Vol. 64, 2011, pp. 204-213.

[29] Conte, D., and Spencer, D., “Targeting the Martian Moons via Direct Insertion into Mars' Orbit," AIAA/AAS Astrodynamic Specialist Conference, At Vail, CO, Vol. 156, Univelt Inc., 2015, pp. 2389-2406.

[30] Organization, N. A. T., "NATO Glossary of Terms and Definitions," Tech. rep., North Atlantic Treaty Organization, 2013. 
[31] Schaub, H., and Junkins, J., Analytical Mechanics of Space Systems, No. v. 1 in AIAA education series, American Institute of Aeronautics and Astronautics, 2003. URL https://books.google.com/books?id=0PpQAAAAMAAJ

[32] Curtis, H., Orbital Mechanics: For Engineering Students, Aerospace Engineering, Elsevier Science, 2015. 